\title{
Robustness of Airline Route Networks
}

\begin{abstract}
Airlines shape their route network by defining their routes through supply and demand considerations, paying little attention to network performance indicators, such as network robustness. However, the collapse of an airline network can produce high financial costs for the airline and all its geographical area of influence. The aim of this study is to analyze the topology and robustness of the network route of airlines following Low Cost Carriers (LCC) and Full Service Carriers (FSC) business models. Results show that FSC hubs are more central than LCC bases in their route network. As a result, LCC route networks are more robust than FSC networks.
\end{abstract}

Keywords: Airline route network, Complex networks, Network robustness, Flight networks

\section{Introduction}

Air transport is among the most dynamic and toughest competition industries in today's global economy. Liberalization of the airline industry (Goetz and Vowles, 2009) has produced very distinct business models among the airlines (Lohmann and Koo, 2013), with the design of their route networks being a strategic factor, in addition to others such as the cost structure and the ancillaries offered.

Most times airlines make the choice of operating a route based on existing supply and demand volumes, paying little attention to other issues such as the robustness of route network operated by the airline. Network robustness (i.e., network resilience in facing disruptions like node isolation) is considered of secondary importance, although a robust route network can provide great improvement to the stability and security of carrier operations. Many domestic airlines are often associated with the image of a country or region and produce a huge economic impact on their national and international economies. The collapse or a critical error of an airline network can 
produce high financial costs for the airline and throughout its geographical area of influence (Guimerà and Amaral, 2004). For instance, the eruption of volcano Eyjafallajökull on March 14, 2010 in Iceland restricted European air traffic and left areas out of operation for 30 days (Brooker, 2010). It caused losses of about 10 million USD because of delays in the operating airports.

The literature on network robustness makes a distinction between errors, the removal or isolation of one or several nodes picked at random, and attacks, which consist in the removal or isolation of nodes that play a vital role in maintaining a network's connectivity (Albert et al., 2000). The examination of flight networks (Zanin and Lillo, 2013) (i.e., networks where the airports act as nodes and are connected if at least one direct route between them exists) through complex networks techniques can provide a deeper understanding of airline networks' behavior when facing errors and attacks. This analysis can be carried out at different levels of analysis (Lordan et al. 2014a): global or regional, which take into account all connections of a global or regional network, alliance, which considers the routes operated by members of an alliance; and airline, where the routes operated by an airline are considered.

Recent articles have analyzed the topology of the air transport network (mainly described by the degree distribution) to understand their distribution and characteristics, most of them focused on the global Guimerà et al. 2005) and regional (Li and Cai, 2004; Guida and Maria, 2007; Wang et al., 2011; Zeng et al., 2011) levels of analysis. Some studies have optimized the paths of the transportation network (Du et al., 2013; Liu et al., 2014) using techniques such as particle swarm optimization (Du et al., 2015; Gao et al. 2015). Other studies have analyzed the robustness of air transport network in order to determine which airports can be considered critical if they were to cease operations. That stream of research considers the regional (Lacasa et al., 2009; Liu et al., 2011) or global (Lordan et al., 2014b) levels of analysis. Finally, Cento (2009); Reggiani et al. (2010) and Lordan (2014) have addressed the study of route networks of individual airlines, and Lordan et al. (2015) has analyzed the robustness of airline alliance route networks. But no study, to our knowledge, has attempted to assess the robustness of airline route networks.

The aim of this study is therefore to analyze the robustness of route networks of airlines which are following different business models and facing isolation of nodes picked at random (errors) or the isolation of nodes 
chosen using a node selection criterion (attacks). In order to compare the robustness of the point-to-point and hub-and-spoke network configurations the set of airlines to be studied will include Full-Service Carriers and Low-Cost Carriers.

\section{The influence of airline business models on route network con- figuration}

Currently, there are two predominant business models in the airline industry: Full-Service Carriers (FSC) and Low-Cost Carriers (LCC). These types of carriers are characterized by having, respectively, hub-and-spoke (HS) and point-to-point (PP) network configurations. Both HS and PP configurations can be described schematically through a network of four nodes, as shown on Figure 1. The HS configuration (right) consists of a central node or hub $\mathrm{H}$ connected to the other nodes, thus only three routes are needed to bond the four destinations. In this configuration the central nodes of the route network are determinant to network resilience when facing node isolation (OKelly, 2014). The PP configuration (left) uses a total of 6 routes to connect all possible node pairs. Generalizing these concepts for $n$ airports to connect all their destinations, the PP configuration requires up to $n(n-1) / 2$ routes (some of which might not be present if they are not profitable enough) while the basic HS configuration works with only $n-1$ routes Alderighi et al., 2007). It is also important to consider the temporal distribution of each model, a fact directly related to the schedule of flights for each airline. The hub of the HS configuration causes the concentration of high traffic density in space and time (Reynolds-Feighan, 2001).

Arlines' business models and strategies strongly define their network structure. Traditional airline network analysis measures the topology variables depending on traffic distribution or concentration of frequencies (Bowen, 2002, McShan, 1986; Reynolds-Feighan, 2001). One objective of these studies is to relate, compare and resemble an airline network to the HS and PP configurations. LCC adopt a PP network configuration because they connect city pairs that offer high load factors and therefore optimize their operability. On the other hand, FSC often develop a HS configuration, offering more destinations by using one or more strategic hubs where large passenger flows concentrate. This allow FSC to get a profitable load factor on routes applying economies of scale (Alderighi et al., 2007). 
As can be seen in Figure 1, the HS configuration concentrates a larger volume of flights and passenger traffic in the switching times. For example, to be able to go from $\mathrm{B}$ to $\mathrm{A}$ and from $\mathrm{C}$ to $\mathrm{A}$ one must call at $\mathrm{H}$. Therefore, it is necessary to coordinate the arrivals of the first section and the exit of the second at the hub. This would give enough time to operate the connection without hindering the operability of the airline. The PP traffic model is temporally and spatially more dispersed because airlines adopting this configuration often operate from airports where planes stay over. The reasons for this strategy can be a lack of demand on certain routes, the absence of slots at airports and rotation needs in order to optimize the operational fleet (Alderighi et al., 2007).

However, the design of an airline network is a complex process adapted to maximize the profitability of the airline. Thus, it is not surprising that the FSC and LCC business models evolve depending on market opportunities. For example, in 2006 Iberia created Clickair as a LCC following a multi-brand strategy to maintain product differentiation. That same year Aer Lingus was redefined as a LCC because of the major survival threats it was facing (Cento, 2009). However, robustness issues, although not usually considered in airline network development, can seriously affect airline profitability in the long term and have a marked impact on the economy.

The way that airlines configure their route network has an impact on its robustness in facing airport closure since the robustness of complex networks is highly dependent on network topology (Albert et al., 2000). On the one hand scale-free networks, which grow through preferential attachment (new nodes are more likely to link to high connected links) and with a power law degree distribution, are resilient to errors and fragile to attacks. On the other hand random networks, where any two nodes can be connected with equal probability and with a Poisson degree distribution, are more resilient to attacks but less resilient to errors than scale-free networks. A real airline route network might fall somewhere between the scale-free and random network configurations, thus determining its robustness in facing airport closure. Thus a study of the topology of the airline route networks can give insights into its robustness in facing airport closure.

\section{Methods}

The route networks of 10 FSCs and 3 LCCs (see Table 1) have been analyzed. The sample of airlines includes FSCs belonging to the main three cur- 
rent airline alliances (i.e., Star Alliance, oneworld and SkyTeam) fulfilling the requirements of a maximum number of airports, a maximum number of passengers per year and/or maximum income within their alliance as published respectively in their annual report for 2012 (Star Alliance, 2013; oneworld, 2013; SkyTeam, 2013). The sample is completed by three airlines known as representatives of the low-cost business model. airberlin was considered a LCC before becoming part of oneworld but in this study has been considered as a FSC. This is because it belongs to an airline alliance and cooperates with other airlines, a feature uncommon for a LCC. The sample is completed by the 3 LCC with the highest number of flights per year: Ryanair, easyJet and Southwest Airlines. The selected sample includes airlines from North America, Europe and China with business models clearly identifiable with FSC and LCC, and airlines that can have a hybrid business model (Klophaus et al., 2012), like airberlin.

The month of August is the period with the highest passenger traffic which directly affects passengers relocation, and is the month with the highest contribution margins for the airlines. The route networks for scheduled flights of August 2013 have been chosen for the analysis in order to be able to assume this analysis as static without taking into account the relocation of passengers. Air routes data have been obtained from the Schedule Reference Service (SRS), a neutral database of scheduled flights complied by IATA (http://www.iata.org/publications/srs/Pages/index.aspx).

The unweighted, undirected graph of the route network for each airline has been constructed for the period considered. Graph nodes represent airports, and two nodes are connected if the airline schedules at least one direct flight between them. The network is treated as undirected since just a small number of flights follow a "circular" pattern (Guimerà et al., 2005). Only flights that are operated for the selected airlines are being considered and therefore the flights operating under codesharing agreements are not considered. The aim of this study is to analyze the robustness of the airline so adding codesharing flights could blur the results. For example, by considering the codesharing flights on the network of British Airways, Dallas/Fort Worth would appear as the airport with the highest degree, followed by London Heathrow, O'Hare, Miami and London Gatwick airports. However BA is actually not allowed to flight a route from Dallas/Fort Worth to another American destination. If this airport had been selected the resulting robustness would have been miscalculated. Disconnecting their real hubs (i.e., 
London Heathrow, London Gatwick and London City) BA would not have been able to fly any route from Dallas/Fort Worth or O'Hare or Miami airports. Table 2 shows the list of selected airlines together with the number of airports $(N)$, number of connections $(E)$ and other topological properties of each network.

Network robustness can be assessed through the effect of the isolation of a fraction $f$ of nodes on the size of the network's giant component (ie, the connected subset of the network with the highest number of nodes). The network will be robust when the size of the giant component decreases little for relatively high values of $f$ (Petreska et al., 2010; Mishkovski et al., 2011). The nodes to isolate can be selected either at random (errors) or through a criterion usually based on node centrality (attacks) (Albert et al., 2000) The study of the robustness allows to evaluate the capacity of a network to avoid a malfunction when a fraction of its components is damaged (Boccaletti et al., 2006).

To simulate errors, 1,000 iterations of random airport closures for each airline were simulated. To simulate attacks, the importance of each airport according to a measure of centrality has been determined and then the airport with the highest value of that centrality has been isolated. After each disconnection, the centralities are recalculated so that the next attack strikes the new most central airport. Two standard measures of node centrality in networks have been used: degree and betweenness. Both measures are computed from the adjacency matrix $\mathcal{A}$. For each pair $(i, j)$ of airports connected by at least one route the element of the adjacency matrix $a_{i j}$ equals 1 , and 0 otherwise.

The degree $k_{i}$ of a node $i$ is the number of edges incident with the node, and is defined as:

$$
k_{i}=\sum_{j} a_{i j}
$$

For a airline route network the degree equals the number of destinations operated from airport $i$ by the airline. The histogram of node degree in the graph allows for the assessment of the degree distribution. The network is scale-free if the degree distribution follows a power law. The betweenness $b_{i}$ of a node $i$ is the number of times that a node appears between the shortest paths of two other nodes: 


$$
b_{i}=\sum_{i \neq j} \frac{n_{j k}(i)}{n_{j k}}
$$

where $n_{j k}$ is the number of shortest paths connecting $j$ and $k$, while $n_{j k}(i)$ is the number of shortest paths connecting $j$ and $k$ and passing through $i$.

\section{Results}

\subsection{Network topology}

Considering the impact of network topology on robustness, an analysis of degree distributions of the airline network for the selected airlines has been conducted. As for degree correlations all route networks are dissortative: airports with low degree are more likely connected with highly connected ones (Boccaletti et al., 2006). Figure 2 shows the cumulative degree distribution plotted in double-logarithmic scale where $P(k)$ cum is the cumulative probability for a node to have degree $k$. There are three different behaviors present and none of them follows a Poisson distribution as would be expected for random graphs. This simple interpretation allows for a distinction between the topological differences of the business models.

At the top of Figure 2 there are the curves of the route networks of the LCC: Ryanair, easyJet and Southwest Airlines. They have the lowest initial gradient and are concave in shape. This means that these carriers will have a more uniform network connection distribution. This shallow slope highlights the presence of a high number of airports with 10 or more connections. This is the characteristic behavior of the PP configuration which leads these airlines to have the highest values of mean degree $\langle k\rangle$ of the sample (see Table 2). Despite having this configuration, LCCs also have operating airport bases with a high number of connections which they use for operating and maintenance purposes. For instance, Ryanair has around $50 \%$ of its airports with ten or more connections while already having 115 connections in London Stansted.

At the bottom of Figure 2 the degree distributions of FSCs' route networks can be found. They have a steeper negative slope than both preceding sets which underlines the existence of many airports with 1 to 5 connections and very few with over 100 connections. For instance, the network of British 
Airways consists of 186 airports spread over the five continents. London Heathrow the node with the largest degree in British Airways network with 125 destinations, followed by London Gatwick with 42 and London City with 20. The connections of the rest of the airports are drastically lower: around $75 \%$ of airports have just one connection, therefore their values of $\langle k\rangle$ are the lowest in Table 2 .

Between the curves of LCCs and FSCs stand the curves of airberlin, China Eastern and China Southern route networks. The degree distribution of the route networks of these airlines follow a more linear distribution in a doublelogarithmic scale. Their mean degree values stand between values of the LCCs and FSCs, as shown in Table 2. Therefore, these carriers stay between the LCCs and the remaining airlines, all of them FSCs. This suggests that their route network design stands in the middle of the PP and HS, and provides evidence that the business model of these airlines stands in the middle of the traditional business models. These airlines are a case of LCCs going hybrid, as suggested in Klophaus et al. (2012).

\subsection{Robustness}

Figure 3 shows the evolution of the size of giant component as a function of the fraction $f$ for isolated airports as a result of the three criterion of node isolation: random, degree and betweenness.

The route networks of all the airlines in the sample react in a similar way facing a random isolation of nodes (see the point-dotted line in Figure 3). The decrease of the giant component for $f=0.05$ ranges from 5 to $10 \%$ of the initial size. Although there are not major differences between FSC and LCC behaviors against errors, some minor differences can be observed. Reviewing the decrease caused by a $f=0.05$ disconnection, LCC ranges from $5-5.5 \%$ while FSC ranges from $7-10 \%$. FSC are a little less resilient than LCC against random isolation of nodes.

Figure 3 also shows that the most effective criterion for attack simulation is betweenness centrality. But in some cases there are small differences between the results obtained with the degree and betweenness criteria because of the network size and its structure. These are networks that are relatively small with a size of between 86 to 362 airports (see Table 2), where the airport that has the largest number of routes is usually the busiest. For Lufthansa, US Airways, American Airlines and British Airways the giant component 
variation is identical for both criteria. The airlines with the most distinct degree and betweenness curves are China Eastern and Ryanair. For China Eastern, for $f=0.104$ the giant component of the degree has 31 more nodes than the one of betweenness. For Ryanair, the maximal difference between criteria is 21 nodes for $f=0.270$.

A comparison of the robustness of sampled airlines is shown both with the range of values of $f$ before networks collapse (Figure 4 a), and for the lowest values of $f$ (Figure $4 \mathrm{~b}$ ) to achieve a better insight to the size reduction of the giant component after disconnecting the first airports. Since the betweenness criterion has turned out to be the most effective, it will be the one considered from now on. By analyzing the behavior of the airlines against attacks a clear difference in the resilience of different business models is observed, which groups once again airlines into three subsets.

In Figure 4 a it can be observed that LCCs are much more robust and have a higher tolerance to attacks than FSCs. For LCCs, to have less than 5\% of the network connected is necessary to disconnect up to a routes fraction of 0.28 for Ryanair and 0.26 for Southwest Airlines. For easyJet the fraction of nodes to delete is lowered to 0.17. It can also be observed that at the start of the disconnection process (Figure $4 \mathrm{~b})$ the LCC also behave with a higher robustness. After removing a fraction of nodes $f=0.04$, the giant component of the network of Southwest Airlines, Ryanair and easyJet still have $91 \%, 84 \%$ and $70 \%$ of the initial size, respectively. Southwest's air route network is the most robust, although it has the lowest number of airports, as airline network robustness is not proportional to network size but its intrinsic structure.

In consequence with Figure 4 it is considered that Ryanair and Southwest fall into the first of the three categories introduced above. As can be observed in Figure 4a, Southwest is initially more robust than Ryanair, while when for $f>11 \%$ the latter reaches the first place in the robustness rank. Despite the fact of also being a LCC, easyJet demonstrates similar behavior to China Eastern, China Southern and airberlin.

According to what has been shown in the degree distribution section, China Eastern (MU), China Southern (CZ) and airberlin (AB) show a particular behavior as FSC both on the global range (Figure 4 a $)$ of disconnection and on the initial region (Figure $4 \mathrm{~b}$ ). In order to have the network almost disconnected, i.e. just $5 \%$ of its original size, these airlines must have a frac- 
tion of their airports disconnected closer to easyJet than to the other FSC, and have values of $f=0.13(\mathrm{MU}), 0.14(\mathrm{CZ})$ and $0.12(\mathrm{AB})$. For $f \sim 0.04$ the network size is much higher than it is for the other three carriers being $56 \%$, $46 \%$ and $48 \%$ respectively. This can be interpreted as the result of a network structure laying somewhere in between the PP and HS. Although it is true that each airline has one or two central airports both in terms of degree and in betweenness measures (Shanghai and Kunming for MU, Dusseldorf and Berlin-Tegel for $\mathrm{AB}$ and Guangzhou for $\mathrm{CZ}$ ) the rest of the network has a structure similar to a PP. The size of the giant component against attacks is substantially more gradual than in the HS configuration.

The last category of airlines includes Delta, British Airways, United Airlines, US Airways, Lufthansa and American Airlines. These are the airlines where the isolations to central nodes have greater impact. For $f \simeq 0.04$ the size of their network plunges to under $5 \%$ of the initial size generating a severe state of operativity lack (Figure $4 \mathrm{~b})$. This behavior can be associated to the HS structure as, once the most central nodes are attacked, the network is almost completely disconnected. Given the rapid pace of network breakage, in this case it is important to assess the damage to lower fractions. For $f=0.01$, the decrease in size is not uniform and varies in relation to the existence of a single hub or more (i.e., multi-hub-and-spoke configuration).

From the results, it can be concluded that the FSC have a network configuration that makes them weaker against the isolation of central nodes. For example, Charlotte-Douglas, Phoenix and Philadelphia are US Airways' three main airports. They are followed by a less central airport but with a big difference in centrality from the fifth, Washington-National airport. After attacking the first two $(f=0.01)$ the size of the giant component is reduced to $64 \%$, maximum robustness in this category, but after attacking the third and fourth $(f=0.02)$ it is reduced to just $6 \%$. On the other extreme there is British Airways which, as discussed above, centralizes its operations at Heathrow, followed by Gatwick and London City. By removing only a fraction $f=0.01$ (i.e. the first two airports) the size of the giant component falls to $12 \%$ of its initial value, the minimum robustness of this category. For $f=0.02$, after closing the third and forth airports, the size drops to $5 \%$. By also considering that an incident affecting Heathrow would suppose the closure of the nearby airspace and consequently all the airports in London, the network of British Airways would be virtually disconnected and practically inoperative with a single incident affecting London air space. 
The response to attacks of Delta, the airline with the largest route network is also noteworthy. After removing its four most central airports, Atlanta, Minneapolis St. Paul, Detroit and New York JFK its network still has half of its nodes connected. Atlanta stands out significantly in terms of degree and betweenness while the other three airports have similar but lower indices. After the isolation of the next three airports reaching $f=0.02$, the size falls to $23 \%$. Finally it is important to highlight Air France's robustness which after falling sharply to $f=0.01$ with the disconnection of its main hub ParisCharles De Gaulle the size is lowered to $38 \%$. However, subsequent isolation of Paris-Orly and the other airports downsize in a much more gradual way since the degree and betweenness indices decrease at a significantly lower rate than the other airlines. The network that remains after removing ParisCharles De Gaulle has a structure similar to a PP configuration, thus leaving a network structure similar to airberlin, China Eastern and China Southern but with a much more prominent central hub.

Such considerations can determine that the damage caused by intentional attacks to networks with an HS configuration is higher than to those with a $\mathrm{PP}$ configuration. The more important the hubs are in the route network the more severe the damage that results from their isolation. The fact that hubs are located closer geographically can increase the potential damage, because of the damage associated with the disconnection of the nearby airspace as exemplified by Wilkinson et al. (2011).

\section{Conclusions}

In this article the analysis of the network robustness based on error and attack simulation is performed. An airline sample of thirteen airlines representing the existing business models in the airline industry route network configuration so that differences between different business models on network robustness can be taken into account.

The analysis has confirmed that Full Service Carriers (FSC) adopting a hub-and-spoke (HS) route network are more sensitive to closure of central airports (i.e., with high betweenness centrality) than Low Cost Carriers (LCC) adopting a point-to-point (PP) route network. That is, disconnecting the hubs of an FSC with HS route network causes greater harm than the disconnection of the base airports of a LCC adopting a PP network. As a consequence, Southwest Airlines and Ryanair, the most important LCC at 
the moment turned out to be the airlines with the most robust route network against attacks. Within the set of FSC there are three airlines with unusual behavior. These are China Eastern and China Southern (the only ones in regions outside of Europe or North America) and airberlin, which was an LCC before it joined oneworld. These airlines adopt a hybrid business model which leads them to build a more robust route network than the FSCs, but less than LCCs.

Whereas Figure $4 \mathrm{~b}$ grants a more realistic approach to the isolation of the most central airports, Figure 4 allows for the evaluation of the consequences of disconnecting a higher number of airports. Comparing the robustness of the set of studied airlines, it can be concluded that it is possible to attenuate the consequences of attacks by means of a multi-hub-and-spoke network. This network should be built by hub airports strategically placed at a distance from each other in order to avoid larger problems related to airspace closure. So, the spatial nature of air route networks has to be taken into account to increase network robustness (Ducruet and Beauguitte, 2013). This distance would allow for homogenization of the importance and centrality of the network without either losing operability or the possibility of applying economies of scale. A second action to increase robustness would be to schedule PP connections in the less central airports and to connect them if one of their hubs fails, thus achieving with this a structure somewhere between HS and PP. This second solution should take into account the supply and demand of each route in order not to hinder airlines' profitability.

It is worth mentioning that these considerations are based on topological criteria applied to complex networks. In the day to day running of airlines there are many other aspects that define airline strategies (Daft and Albers, 2013), that can have an impact on route structure and network strategy. Therefore the application of the proposed solution has to be evaluated in depth and in detail. In future studies the passenger flux could be taken into account and, as a result, an interpretation of the traffic loss caused by the closure of airports may arise. Passenger flows are also central to assess other kinds of disruptions, such as demand vulnerabilities (Connors and Watling, 2014), that can be tackled via hub flow optimization (OKelly, 2010). A similar result would be expected because most central airports are those that concentrate a higher volume of aerial traffic in terms of flights and passengers. Further studies in this area would a complement the evaluation of protection, stability and safety of each airline network according to their 
business model.

\section{Acknowledgement}

Authors would like to thank Associate Professor Wen-Bo Du (Beihang University) for supplying helpful suggestions and comments.

\section{References}

Albert, R., Jeong, H., Barabási, A.L., 2000. Error and attack tolerance of complex networks. Nature 406, 378-82. URL: http://www.ncbi.nlm. nih.gov/pubmed/10935628, doi:10.1038/35019019.

Alderighi, M., Cento, A., Nijkamp, P., Rietveld, P., 2007. Assessment of new hub-and-spoke and point-to-point airline network configurations. Transport Reviews 27, 529-549.

Boccaletti, S., Latora, V., Moreno, Y., Chavez, M., Hwang, D., 2006. Complex networks: Structure and dynamics. Physics Reports 424, 175-308. URL: http://linkinghub.elsevier.com/retrieve/pii/ S037015730500462X, doi:10.1016/j.physrep.2005.10.009.

Bowen, J., 2002. Network change, deregulation, and access in the global airline industry. Economic Geography 78, 425-439.

Brooker, P., 2010. Fear in a handful of dust: aviation and the icelandic volcano. Significance 7, 112-115.

Cento, A., 2009. The Airline Industry. Contributions to Economics, PhysicaVerlag HD, Heidelberg. URL: http://www.springerlink.com/index/ 10.1007/978-3-7908-2088-1, doi:10.1007/978-3-7908-2088-1.

Connors, R.D., Watling, D.P., 2014. Assessing the Demand Vulnerability of Equilibrium Traffic Networks via Network Aggregation. Networks and Spatial Economics URL: http://link.springer.com/10. 1007/s11067-014-9251-9, doi:10.1007/s11067-014-9251-9.

Daft, J., Albers, S., 2013. A conceptual framework for measuring airline business model convergence. Journal of Air Transport Management 28, $47-54$. 
Du, W., Gao, Y., Liu, C., Zheng, Z., Wang, Z., 2015. Adequate is better: particle swarm optimization with limitedinformation. Applied Mathematics and Computation 268, 832838. URL: http://dx.doi.org/10.1016/j.amc.2015.06.062http: //linkinghub.elsevier.com/retrieve/pii/S0096300315008437, doi: $10.1016 / j$.amc.2015.06.062.

Du, W., Wu, Z.X., Cai, K.Q., 2013. Effective usage of shortest paths promotes transportation efficiency on scale-free networks. Physica A: Statistical Mechanics and its Applications 392, 3505-3512. URL: http:// linkinghub.elsevier.com/retrieve/pii/S0378437113002653, doi:10. $1016 / \mathrm{j}$.physa.2013.03.032.

Ducruet, C., Beauguitte, L., 2013. Spatial Science and Network Science: Review and Outcomes of a Complex Relationship. Networks and Spatial Economics 14, 297-316. URL: http://link.springer.com/10.1007/ s11067-013-9222-6, doi:10.1007/s11067-013-9222-6.

Gao, Y., Du, W., Yan, G., 2015. Selectively-informed particle swarm optimization. Scientific Reports 5, 9295. URL: http://www.nature.com/ doifinder/10.1038/srep09295, doi:10.1038/srep09295.

Goetz, A.R., Vowles, T.M., 2009. The good, the bad, and the ugly: 30 years of us airline deregulation. Journal of Transport Geography 17, 251-263.

Guida, M., Maria, F., 2007. Topology of the Italian airport network: A scalefree small-world network with a fractal structure? Chaos, Solitons \& Fractals 31, 527-536. URL: http://linkinghub.elsevier.com/retrieve/ pii/S0960077906001792, doi:10.1016/j.chaos.2006.02.007.

Guimerà, R., Amaral, L.a.N., 2004. Modeling the world-wide airport network. The European Physical Journal B - Condensed Matter 38, 381-385. URL: http://www.springerlink.com/ openurl . asp?genre=article\&id=doi:10.1140/epjb/e2004-00131-0, doi:10.1140/epjb/e2004-00131-0.

Guimerà, R., Mossa, S., Turtschi, A., Amaral, L.a.N., 2005. The worldwide air transportation network: Anomalous centrality, community structure, and cities' global roles. Proceedings of the National 
Academy of Sciences of the United States of America 102, 77949. URL: http://www.pubmedcentral.nih.gov/articlerender.fcgi? artid=1142352\&tool=pmcentrez\&rendertype=abstract, doi:10.1073/ pnas.0407994102.

Klophaus, R., Conrady, R., Fichert, F., 2012. Low cost carriers going hybrid: Evidence from europe. Journal of Air Transport Management 23, 54-58.

Lacasa, L., Cea, M., Zanin, M., 2009. Jamming transition in air transportation networks. Physica A: Statistical Mechanics and its Applications 388, 3948-3954. URL: http://linkinghub.elsevier.com/retrieve/ pii/S0378437109004361, doi:10.1016/j.physa.2009.06.005.

Li, W., Cai, X., 2004. Statistical analysis of airport network of China. Physical Review E 69, 1-6. URL: http://link.aps.org/doi/10.1103/ PhysRevE.69.046106, doi:10.1103/PhysRevE.69.046106.

Liu, C., Du, W., Wang, W.X., 2014. Particle swarm optimization with scalefree interactions. PLoS ONE 9, 1-8. doi:10.1371/journal . pone.0097822.

Liu, H., Hu, X.B., Yang, S., Zhang, K., Di Paolo, E., 2011. Application of Complex Network Theory and Genetic Algorithm in Airline Route Networks. Transportation Research Record: Journal of the Transportation Research Board 2214, 50-58. doi:10.3141/2214-07.

Lohmann, G., Koo, T.T., 2013. The airline business model spectrum. Journal of Air Transport Management 31, 7-9.

Lordan, O., 2014. Study of the full-service and low-cost carriers network configuration. Journal of Industrial Engineering and Management 7, 1112-1123. URL: http://www.jiem.org/index.php/jiem/article/ view/1191, doi:10.3926/jiem.1191.

Lordan, O., Sallan, J.M., Simo, P., 2014a. Study of the topology and robustness of airline route networks from the complex network approach: a survey and research agenda. Journal of Transport Geography 37, 112-120. URL: http://linkinghub.elsevier.com/retrieve/pii/ S0966692314000763, doi:10.1016/j.jtrangeo.2014.04.015. 
Lordan, O., Sallan, J.M., Simo, P., Gonzalez-Prieto, D., 2014b. Robustness of the air transport network. Transportation Research Part E: Logistics and Transportation Review 68, 155-163. URL: http://linkinghub. elsevier.com/retrieve/pii/S1366554514000805, doi:10.1016/j.tre. 2014.05.011.

Lordan, O., Sallan, J.M., Simo, P., Gonzalez-Prieto, D., 2015. Robustness of airline alliance route networks. Communications in Nonlinear Science and Numerical Simulation 22, 587 - 595. URL: http:// linkinghub.elsevier.com/retrieve/pii/S1007570414003529, doi:10. $1016 /$ j.cnsns.2014.07.019.

McShan, W.S., 1986. An economic analysis of the hub-and-spoke routing strategy in the airline industry. Transportation Center, Northwestern University.

Mishkovski, I., Biey, M., Kocarev, L., 2011. Vulnerability of complex networks. Communications in Nonlinear Science and Numerical Simulation 16, 341-349. URL: http://linkinghub.elsevier.com/retrieve/pii/ S1007570410001607, doi:10.1016/j.cnsns.2010.03.018.

oneworld, 2013. oneworld at a glance. http://www.oneworld.com/ news-information/oneworld-fact-sheets/. Accessed: 2014-11-27.

OKelly, M.E., 2010. Routing Traffic at Hub Facilities. Networks and Spatial Economics 10, 173-191. URL: http://link.springer.com/10.1007/ s11067-008-9061-z, doi:10.1007/s11067-008-9061-z.

OKelly, M.E., 2014. Network Hub Structure and Resilience. Networks and Spatial Economics , 1-17URL: http://link.springer.com/10.1007/ s11067-014-9267-1, doi:10.1007/s11067-014-9267-1.

Petreska, I., Tomovski, I., Gutierrez, E., Kocarev, L., Bono, F., Poljansek, K., 2010. Application of modal analysis in assessing attack vulnerability of complex networks. Communications in Nonlinear Science and Numerical Simulation 15, 1008-1018. URL: http:// linkinghub.elsevier.com/retrieve/pii/S1007570409002639, doi:10. $1016 / j$.cnsns.2009.05.002.

Reggiani, A., Nijkamp, P., Cento, A., 2010. Connectivity and concentration in airline networks: a complexity analysis of Lufthansas network. 
European Journal of Information Systems 19, 449-461. URL: http: //papers.ssrn. com/sol3/Delivery. cfm?abstractid=1908157http: //www . ingentaconnect.com/content/pal/0960085x/2010/00000019/ $00000004 /$ art00008.

Reynolds-Feighan, A., 2001. Traffic distribution in low-cost and full-service carrier networks in the us air transportation market. Journal of Air Transport Management 7, 265-275.

SkyTeam, 2013. SkyTeam \& Members Fact Sheet. http://www.skyteam . com/en/About-us/Press/Facts-and-Figures/. Accessed: 2014-11-27.

Star Alliance, 2013. Star Alliance Facts and Figures Nov 2012. http: //www . staralliance.com/en/about/member_airlines/. Accessed: 2014-11-27.

Wang, J., Mo, H., Wang, F., Jin, F., 2011. Exploring the network structure and nodal centrality of China's air transport network: A complex network approach. Journal of Transport Geography 19, 712-721. URL: http:// linkinghub.elsevier.com/retrieve/pii/S0966692310001328, doi:10. $1016 / j \cdot j$ trangeo.2010.08.012.

Wilkinson, S.M., Dunn, S., Ma, S., 2011. The vulnerability of the European air traffic network to spatial hazards. Natural Hazards 60, 1027-1036. URL: http://www.springerlink.com/index/10.1007/ s11069-011-9885-6, doi:10.1007/s11069-011-9885-6.

Zanin, M., Lillo, F., 2013. Modelling the air transport with complex networks: A short review. The European Physical Journal Special Topics 215, 5-21. URL: http://link.springer.com/10.1140/epjst/ e2013-01711-9, doi:10.1140/epjst/e2013-01711-9.

Zeng, X., Tang, X., Jiang, K., 2011. Empirical Study of Chinese Airline Network Structure Based on Complex Network Theory. Journal of Transportation Systems Engineering and Information Technology 11, 175-181. URL: http://linkinghub.elsevier.com/retrieve/pii/ S1570667210601572, doi:10.1016/S1570-6672(10)60157-2. 


\begin{tabular}{|c|lll|}
\hline & Airline Name & Alliance & Region \\
\hline LH & Lufthansa & Star Alliance & Europe \\
UA & United Airlines & Star Alliance & North America \\
US & US Airways & Star Alliance & North America \\
AB & airberlin & oneworld & Europe \\
AA & American Airlines & oneworld & North America \\
BA & British Airways & oneworld & Europe \\
AF & Air France & SkyTeam & Europe \\
MU & China Eastern & SkyTeam & China \\
CZ & China Southern & SkyTeam & China \\
DL & Delta & SkyTeam & North America \\
FR & Ryanair & LCC & Europe \\
U2 & easyJet & LCC & Europe \\
WN & Southwest Airlines & LCC & North America \\
\hline
\end{tabular}

Table 1: Airlines analyzed by IATA code 


\begin{tabular}{|c|ccccc|}
\hline & $N$ & $E$ & $\langle k\rangle$ & $L$ & $C$ \\
\hline FR & 178 & 1,396 & 15.69 & 2.16 & 0.44 \\
WN & 86 & 507 & 11.79 & 1.97 & 0.72 \\
U2 & 131 & 601 & 9.18 & 2.19 & 0.39 \\
\hline CZ & 178 & 576 & 6.47 & 2.45 & 0.62 \\
MU & 182 & 571 & 6.27 & 2.5 & 0.55 \\
AB & 119 & 361 & 6.07 & 2.31 & 0.51 \\
\hline DL & 328 & 882 & 5.38 & 2.38 & 0.88 \\
UA & 362 & 933 & 5.15 & 2.57 & 0.91 \\
US & 203 & 408 & 4.02 & 2.26 & 0.96 \\
AA & 272 & 523 & 3.85 & 2.3 & 0.94 \\
LH & 209 & 395 & 3.78 & 2.18 & 0.93 \\
AF & 178 & 258 & 2.9 & 2.42 & 0.46 \\
BA & 186 & 223 & 2.4 & 2.87 & 0.15 \\
\hline
\end{tabular}

Table 2: Main topological properties of airlines route network. The quantities measured are: number of vertices $N$, number of edges $E$, characteristic path length $L$, clustering coefficient $C$ and average degree $\langle k\rangle$. 
Figure 1: HS and PP configurations. Source: (Alderighi et al., 2007)
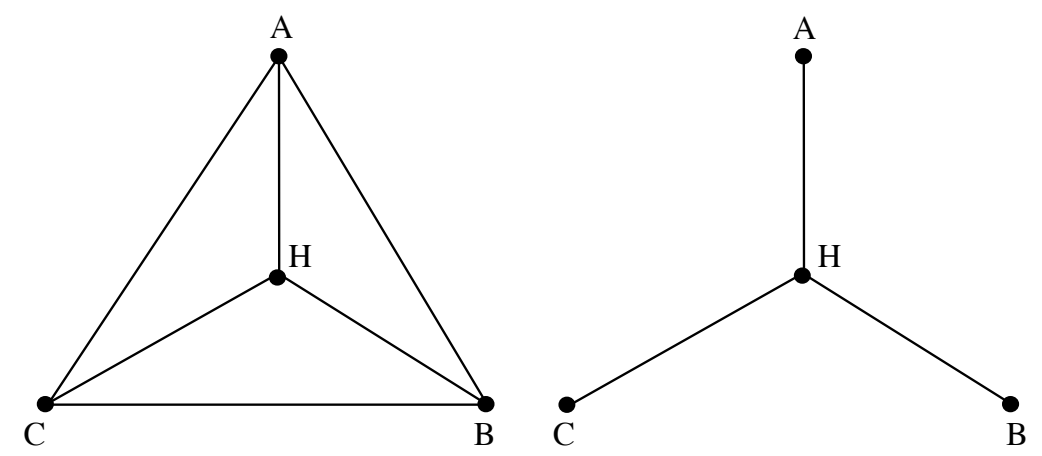
Figure 2: Cumulative degree distribution plotted in double-logarithmic scale

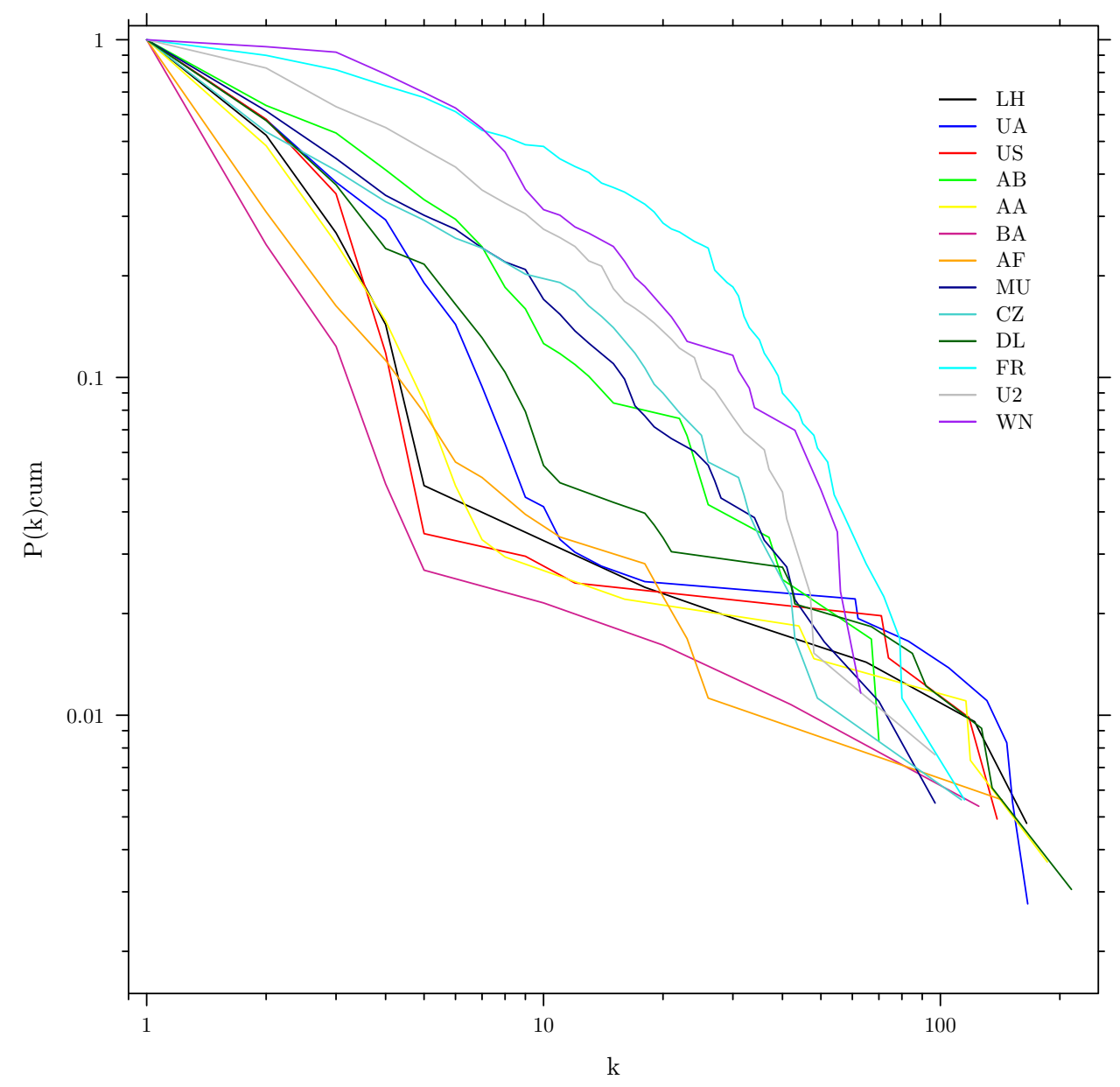


Figure 3: Error and attack vulnerability of each airline transport networks. $y=$ size of the giant component (\%), $x=$ fraction $f$ of disconnected airports (\%). Plain line: betweenness attack, Dotted line: degree attack, Point-dotted line: error
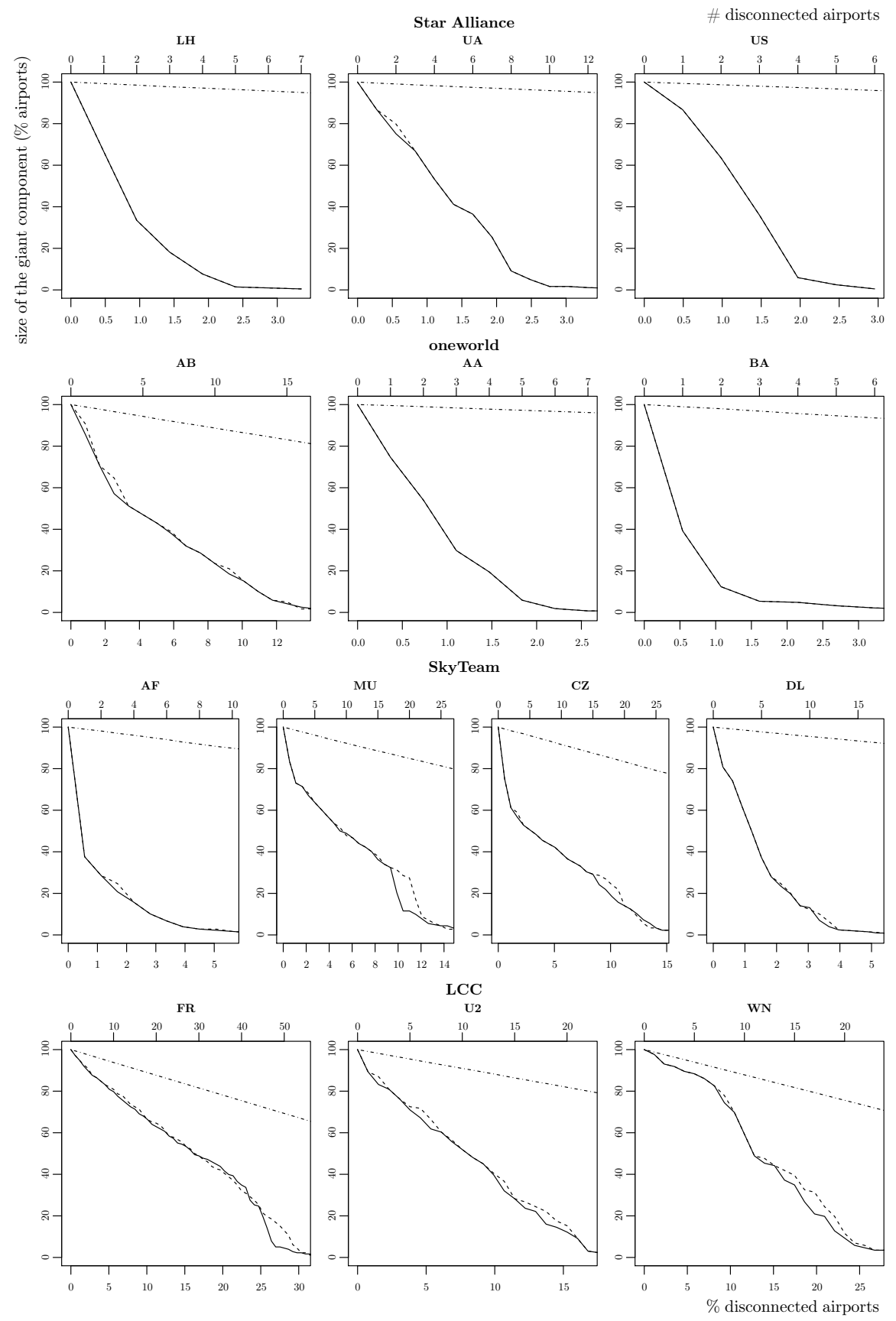
Figure 4: Betweenness attack vulnerability of airline transport networks. $y=$ size of the giant component (\%), $x=$ fraction $f$ of disconnected airports (\%). a. Global $(f \leq 25 \%)$. b. Detail $(f \leq 4 \%)$

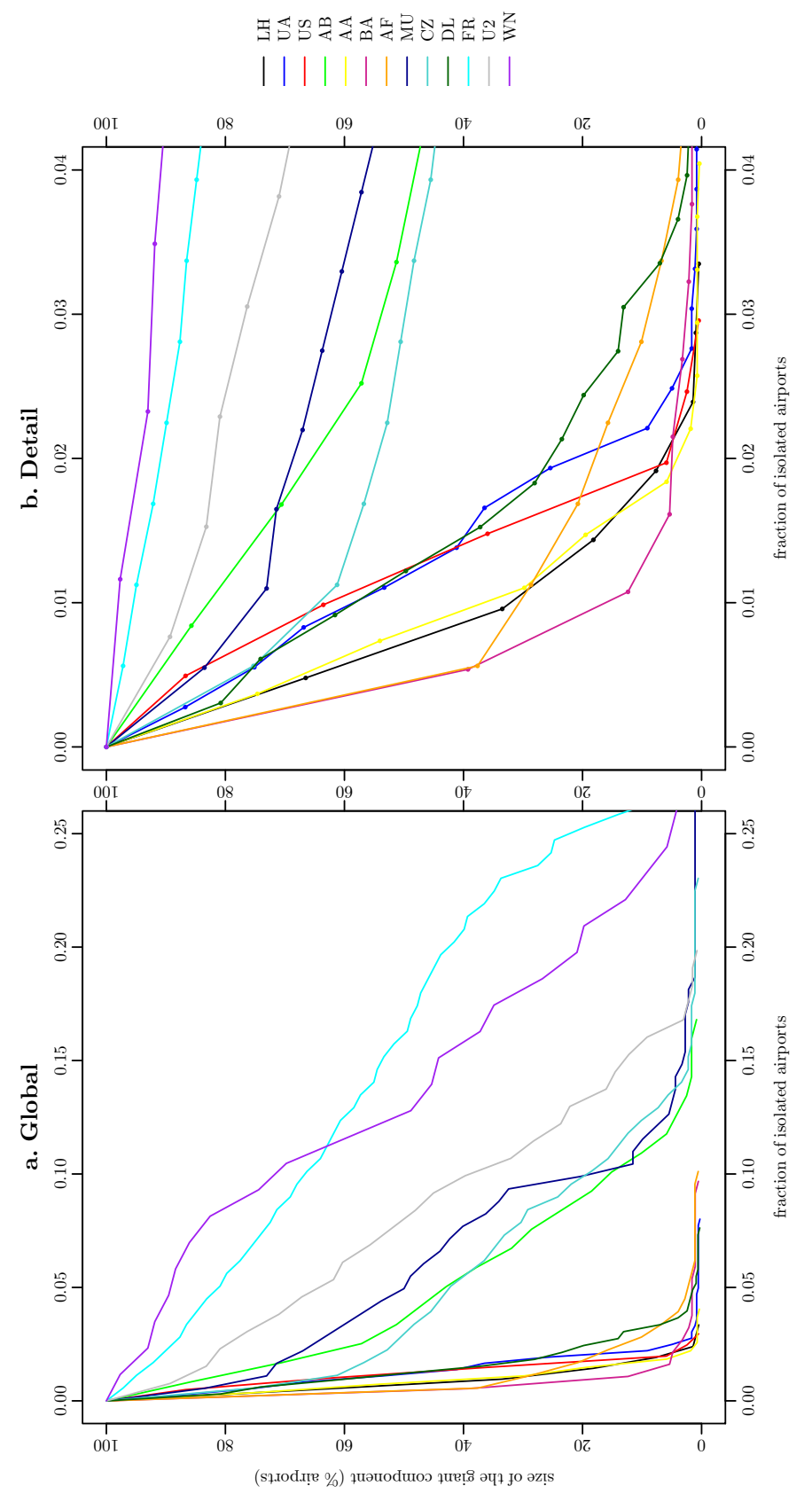

